\title{
Krabbe disease: An unusual presentation of optic nerve enlargement
}

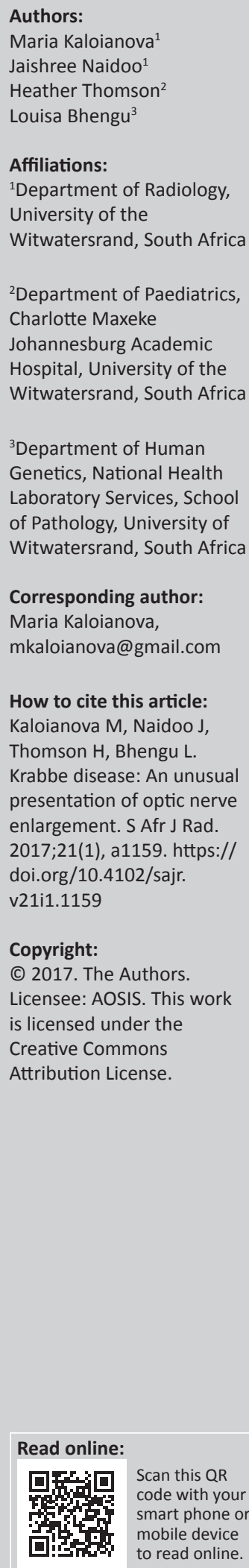

Krabbe disease is an autosomal recessive leukodystrophy that presents clinically with regression of milestones, excessive irritability and inconsolable crying. The pathologic basis of the disease is abnormal myelin metabolism resulting from a deficiency in the galactocerebrosidase enzyme with subsequent white matter destruction. Although optic atrophy is a classic presentation of Krabbe disease, we report on two patients who are biological brothers presenting with optic nerve enlargement in addition to other typical magnetic resonance imaging features of Krabbe disease, thereby confounding the initial diagnosis.
Note: A selection of conference abstracts: RSSA/SASPI Paediatric Imaging Congress, 03-06 November 2016, Spier Estate, Stellenbosch, South Africa. Faculty collaborators: Professor Kassa Darge (Body Imaging, University of Pennsylvania, Philadelphia, USA), Professor Edward Lee (Thoracic Imaging, Harvard University, USA), Professor Beverley Newman (Cardiac Imaging, Stanford University, California, USA), Professor Kimberly Applegate (Image Gently and Body Imaging, Emory University, Atlanta, USA) and Professor Savvas Andronikou (Thoracic Imaging, University of Bristol, UK) supported by South African Paediatric Radiologists, co-ordinated by Dr. Jaishree Naidoo, President of the African Society of Paediatric Imaging and Head of Division of Paediatric Radiology, Charlotte Maxeke Johannesburg Academic Hospital.

This conference abstract is partially based on the following publication: Kaloianova M, Naidoo J, Thomson H, Bhengu L. Krabbe disease An unusual presentation of optic nerve enlargement. S Afr J Rad. 2015;19(2); a887. https://doi.org/10.4102/sajr.v19i2.887 